\title{
Effect of dietary alginic acid on juvenile tilapia (Oreochromis niloticus) intestinal microbial balance, intestinal histology and growth performance
}

\author{
Daniel L. Merrifield • Glenn M. Harper • \\ Sanaa Mustafa - Oliana Carnevali • Simona Picchietti • \\ Simon J. Davies
}

Received: 13 September 2010 / Accepted: 17 December 2010

(C) Springer-Verlag 2011

\begin{abstract}
The aim of the present study was to assess the effect of a commercial alginic acid source (Ergosan) on tilapia Oreochromis niloticus intestinal microbial balance, intestinal morphology, and growth parameters. Fish were fed a basal control diet or the basal diet plus a source of alginic acid (5 $\mathrm{g} \mathrm{kg}^{-1}$ Ergosan; Schering-Plough Aquaculture, UK) for 9 weeks. At the end of the trial, light and electron microscopy demonstrated that the morphology of the intestinal tract at the gross and ultra-structural level was not affected by dietary alginic acid inclusion. Both groups of fish displayed healthy, normal morphology with no signs of disease, cell or tissue damage. Intestinal epithelial leucocyte infiltration was not affected by dietary alginic acid. Molecular bacterial profiles derived from PCR-DGGE illustrated highly similar microbial communities (both within the lumen and associated with the intestinal mucosa) in the respective treatment groups. Microbial ecological
\end{abstract}

\footnotetext{
D. L. Merrifield $(\varangle) \cdot$ S. Mustafa $\cdot$ S. J. Davies

Aquaculture and Fish Nutrition Research Group,

School of Biomedical and Biological Sciences,

The University of Plymouth,

Plymouth, Devon, UK PL4 8AA

e-mail: daniel.merrifield@plymouth.ac.uk

G. M. Harper

Electron Microscopy Centre, University of Plymouth, Plymouth, UK

O. Carnevali

Department of Marine Sciences,

Polytechnic University of Marche,

Ancona, Italy

S. Picchietti

Department Environmental Sciences, Tuscia University,

Viterbo, Italy
}

parameters (e.g. species diversity and richness) also remained unaffected. Although not significant, trends towards elevated survival and body protein content were observed in the alginic acid-fed fish. These results are suggestive that alginic acid does not adversely impact the indigenous gastrointestinal microbial balance and subsequently does not impact upon the epithelial brush border integrity. Validation of non-detrimental impacts of immunostimulatory products on gastric microbiota and epithelial integrity should be pursued in future studies as maintaining microbial balance and epithelial integrity is essential for proper gut functionality.

Keywords Gut microbiota - DGGE · Alginic acid · Ultra histology $\cdot$ Intestine

\section{Introduction}

The use of preventive approaches to combat disease, essential for further development of sustainable aquaculture practices, is becoming increasingly important. Promising among these preventative approaches are immunostimulants. Immunostimulants are compounds able to stimulate the immune response by promoting phagocytic cell function, increasing their bactericidal activity and/or nonspecific cytotoxic cells and antibody production (Sakai 1999). Although treatment and prevention of infectious diseases are the most common reasons to use immunomodulators, amelioration of stress-induced immunosupression and improved general animal well being are also reported benefits (Blecha 2001; Vollostad et al. 2006).

A range of different substances are known to act as immunostimulants but few are suitable for use in aquacul- 